\title{
ASSOCIATION OF A NOVEL POLYMORPHISM OF THE $\beta 2$-CHIMAERIN GENE (CHN2) WITH SMOKING
}

\section{Laura Barrio-Real, PhD ${ }^{1}$, Miguel Barrueco, MD, PhD ${ }^{1}$, Rogelio González-}

Sarmiento, MD, $\mathrm{PhD}^{1 *}$ and María J. Caloca, $\mathrm{PhD}^{2 *}$

${ }^{1}$ Unidad de Medicina Molecular. Facultad de Medicina. Hospital Universitario de Salamanca and IBMCC (CSIC/Universidad de Salamanca). Salamanca. Spain.

${ }^{2}$ Instituto de Biología y Genética Molecular (IBGM), Consejo Superior de Investigaciones Científicas (CSIC), 47003 Valladolid. Spain.

*To whom correspondence should be addressed:

María J. Caloca

Instituto de Biología y Genética Molecular (IBGM), c/ Sanz y Forés s/n, 47003 Valladolid, Spain.

Phone: $\quad+34-983186434$

Fax: $\quad+34-983184800$

E-mail: $\quad$ mj.caloca@ csic.es

Rogelio González-Sarmiento

Departamento de Medicina, Facultad de Medicina, Universidad de Salamanca, C/Donantes de Sangre, s/n 37007 Salamanca, Spain

Phone: $\quad+34923294553$

Fax: $\quad+34923394593$

E-mail: $\quad$ gonzalez@usal.es

Sources of Support: Spanish Ministry of Health (Fondo de Investigaciones Sanitarias, PI052096 to MJC, PI10/00219 to RGS), the Spanish Ministry of Science and Innovation 
cosponsored by the plan E (BFU2009-08051 to MJC), and the Castilla y Leon Autonomous Government (BIO103/VA44/11 to MJC and GR15 to RGS). 


\section{ABSTRACT}

\section{Objective}

The $C H N 2$ gene encodes the $\beta 2$-chimaerin, a Rac specific GTPase activating protein with an important role in the establishment of functional brain circuitry by controlling axon pruning. Genetic studies suggest that the CHN2 gene harbors variants that contribute to addiction vulnerability and smoking behavior. To further evaluate the role of $\beta 2$-chimaerin in nicotine addiction, we investigated the association of three individual polymorphisms of the $C H N 2$ gene with smoking dependence.

\section{Methods}

361 healthy volunteers, 173 smokers (mean age $60.4 \pm 1.4$ ) and 188 control subjects (mean age $45.9 \pm 1.4$ ) were genotyped for three single nucleotide polymorphisms in the CHN2 gene (rs3750103, rs12112301 and rs186911567). The association of these polymorphisms with smoking habits was analyzed.

\section{Results}

There was no significant association of polymorphisms rs12112301 and rs3750103 with smoking. However, there was a significant difference in the frequency of the rs186911567 polymorphism between smokers and controls $(p=0.003)$.

\section{Conclusions}

We report for the first time a significant association of the novel rs 186911567 polymorphism of the $C H N 2$ gene with smoking.

Keywords: $\beta 2$-chimaerin, $C H N 2$, addiction, polymorphisms, smoking. 


\section{INTRODUCTION}

Tobacco addiction is a complex brain disorder that involves multiple molecular components and has a strong genetic influence [1]. Numerous factors contribute to the long lasting nature of tobacco addiction, such as the structural changes induced by nicotine in neurons and its effect on synaptic plasticity [2]. Actin cytoskeleton drives the neuroadaptations produced by nicotine, and therefore, several genes encoding proteins involved in cytoskeleton regulation harbor allelic variants that contribute to smoking dependence [3]. Among these, the CHN2 gene has been identified in genomewide association studies (GWAS) of nicotine dependence as a candidate for harboring variants involved in smoking addiction [4]. The human CHN2 gene maps to chromosome $7 \mathrm{p} 15.3$, has 13 exons and encodes the $\beta 2$-chimaerin, a GTPase activating protein (GAP) highly expressed in the brain [5]. $\beta 2$-chimaerin selectively inactivates Rac, a small GTPase with a prominent role in the control of actin cytoskeleton dynamics [6, 7]. Recent work in animal models has demonstrated the essential role of $\beta 2$-chimaerin in controlling axon pruning in the hippocampus [8]. In humans, the missense polymorphism (H204R) on the CHN2 gene is associated with schizophrenia [9], a disorder with high rate of comorbidity with smoking [10]. Considering the evidences for the involvement of the CHN2 gene in tobacco addiction, we focused this study on identifying the association of individual SNPs on the CHN2 gene with smoking. We analyzed three polymorphisms of potential relevance for $\beta 2$-chimaerin function: polymorphism rs3750103 c.611A $>\mathrm{G}$ (p.H204R) in exon 7 previously associated with schizophrenia, polymorphism rs12112301 (IVS5+7C>T) located on intron 5 that could influence on splicing, and polymorphism rs186911567 c.366G>A (p.S122S) in exon 6, a novel synonymous polymorphism recently identified in our laboratory. 


\section{MATERIALS AND METHODS}

\section{Subjects}

The three SNPs were genotyped in 361 Caucasians from Castilla y León, Spain. 173 participants were current smokers (mean age $60.4 \pm 1.4$ years, $74.0 \%$ males) which fulfilled the DMS-IV criteria for nicotine dependence dictated by the American Psychiatric Association, and were referred to the Tobacco Addiction Unit of the University Hospital of Salamanca, Spain. The control group consisted of 188 subjects (mean age $45.9 \pm 1.4$ years, $75.0 \%$ males) which had a lifetime history of no more than 100 cigarettes without developing an addiction to tobacco. Neither smokers nor controls had any mental disorder, or personal or familiar history of lung cancer. All subjects gave informed consent to participate, and the study was approved by institutional ethical committees.

\section{Genotyping}

Genotypes of the rs12112301 and rs3750103 polymorphisms were determined using the TaqMan 5'-exonuclease allelic distribution assay (Applied Biosystems, Foster City, USA). The rs186911567 polymorphism was studied by PCR-restriction fragment length polymorphism-based analysis and digestion with TaqI. Primers used for the amplification were forward: 5'-TATCATTCCACACTGTGCTTAT-3', and reverse: 5'TCAGCAAATCGCACCTATAGT-3'. The amplified 433 bp fragment was digested overnight at $65{ }^{\circ} \mathrm{C}$ with TaqI and the resulting fragments (298 bp and $135 \mathrm{bp}$ for A allele and $433 \mathrm{bp}$ for $\mathrm{G}$ allele) were separated on a $3 \%$ agarose gel.

\section{Statistical analysis}


Hardy-Weinberg equilibrium was determined with the $\chi^{2}$ test. Genotype frequencies of smokers and controls, and smokers's subgroups, were compared by means of $\chi^{2}$ test and Fisher's exact test when necessary (expected values below 5). $P<0.05$ was considered as significant for the differences between the genotypes. All statistical analyses were performed using the statistical software SPSS v.19.0 (SPSS Inc., Chicago, IL). 


\section{RESULTS}

Table 1 shows genotype distributions of the three polymorphisms of the CHN2 gene among the smokers and controls. Frequencies of the rs12112301 and rs3750103 polymorphisms were in Hardy-Weinberg equilibrium in the control group and no significant differences were observed among smokers and controls. However, we found a significant difference in the frequency of the rs186911567 polymorphism between smokers and controls. $4.6 \%$ of smokers had the GA or AA genotypes while no carriers of the A allele were found in the control group $(p=0.003)$. Hardy-Weinberg equilibrium was not considered for this polymorphism in the control group since only carriers of the GG genotype were found in these subjects.

To determine the factors contributing to the association of the rs 186911567 polymorphism with smoking, we next analyzed this polymorphism in smokers grouped according to their age and smoking habits (Table 2). There was no significant association of this polymorphism with the daily cigarette consumption (up to or more than 20), years of smoking (up to or more than 30) or age (>65 vs. <65). However, there was a trend towards an increased frequency of the polymorphism in older smokers ( $p=0.07) .7 .5 \%$ of smokers older than 65 had the GA or AA genotypes, while only $1.25 \%$ of smokers younger than 65 had the GA genotype and no carriers of the AA genotype were found in this group. These data suggest that the rs 186911567 polymorphism does not influence smoking quantity (estimated by the number of cigarettes smoked per day) but may influence duration of smoking. 


\section{DISCUSSION}

In this study we evaluate for the first time the association of individual SNPs of the $C H N 2$ gene with smoking. Our results are in agreement with the suggested role for the $C H N 2$ gene in addiction. Uhl et al. [4] identified the $C H N 2$ among the genes that facilitate nicotine abstinence in smokers treated with nicotine replacement therapies or bupropion. Our study, however, identify a SNP that contributes to smoking. Thus, our data are consistent with whole genome association studies that identify the CHN2 gene among the 89 genes with variants that contribute to addiction vulnerability [11].

The functional relevance of the synonymous rs186911567 polymorphism is currently unknown. Although the $\mathrm{G} \rightarrow$ A substitution at position 366 in exon 6 does not result in aminoacid change, bioinformatics analysis predicts the disruption of exonic splicing enhancer motifs and loss of a binding site for the splicing factor SC35 (Human Splicing Finder) [12]. Therefore, a theoretical option is that polymorphism rs 186911567 could alter the splicing of the $\beta 2$-chimaerin transcript, rendering an inactive protein by skipping of exon 6 and generation of a premature stop codon. Inactivation of $\beta 2$ chimaerin would result in increased Rac activity and altered actin cytoskeleton remodeling, an important process for the nicotine effect on neural plasticity. Interestingly, chn2 knockout mice show defects in axon and synaptic pruning, processes that are unbalanced in numerous mental disorders [13]. Furthermore, polymorphisms in the NRXN3 gene, important regulator of dendritic and axonal arbors remodeling, have been associated with smoking as well as with schizophrenia $[14,15]$.

Other pathways may also contribute to the role of $\beta 2$-chimaerin on nicotine dependence. Recent data demonstrate that the effects of nicotine on learning and memory are in part mediated by JNK signaling in the hippocampus, which may explain 
the long-lasting modifications of behavior induced by nicotine that contributes to addiction [16]. It is plausible that $\beta 2$-chimaerin regulates JNK activity in hippocampal neurons since it has been reported the expression of this protein in the hippocampus [8] and the role of $\beta 2$-chimaerin in the regulation of JNK has been already demonstrated in epithelial cells [17].

In summary, our study is the first report of a novel SNP polymorphism of the CHN2 gene that may contribute to smoking behavior. Because of the relatively small sample size, this is a preliminary study. However, our results are in line with a role of the $C H N 2$ gene in smoking and support the relevance of this protein in addiction. Based on these findings and the known biological role of $\beta 2$-chimaerin, further studies are needed to corroborate the utility of rs186911567 polymorphism as a genetic marker for smoking dependence. 


\section{Funding}

This work was supported by the Spanish Ministry of Health (Fondo de Investigaciones Sanitarias, PI052096 to MJC, PI10/00219 to RGS), the Spanish Ministry of Science and Innovation cosponsored by the plan E (BFU2009-08051 to MJC), and the Castilla y Leon Autonomous Government (BIO103/VA44/11 to MJC). 


\section{REFERENCES}

1. Munafò MR, Clark TG, Johnstone EC et al. The genetic basis for smoking behavior: A systematic review and meta-analysis. Nicotine \& Tobacco Research. 2004; 6:583-597.

2. Nestler EJ. Molecular basis of long-term plasticity underlying addiction. Nat Rev Neurosci. 2001; 2:119-128.

3. Lind PA MS, Vink JM, Pergadia ML, et al. A genomewide association study of nicotine and alcohol dependence in Australian and Dutch populations. Twin Res Hum Genet. 2010; 13:10-29.

4. Uhl GR. Liu QR, Drgon T, et al. Molecular Genetics of Successful Smoking Cessation: Convergent Genome-Wide Association Study Results. Arch Gen Psychiatry. 2008; 65:683-693.

5. Yuan S, Miller DW, Barnett GH, et al. Identification and characterization of human beta 2-chimaerin: association with malignant transformation in astrocytoma. Cancer Res. 1995; 55:3456-3461.

6. Caloca MJ, Wang H and Kazanietz MG. Characterization of the Rac-GAP (RacGTPase-activating protein) activity of beta2-chimaerin, a 'non-protein kinase $C^{\prime}$ phorbol ester receptor. Biochem J. 2003; 375:313-21.

7. Caloca M.J. Delgado P, Alarcon B, et al. Role of chimaerins, a group of Racspecific GTPase activating proteins, in T-cell receptor signaling. Cellular Signalling. 2008; 20:758-770. 
8. Riccomagno MM., Hurtado A, Wang HB, et al. The RacGAP $\beta 2$-Chimaerin Selectively Mediates Axonal Pruning in the Hippocampus. Cell. 2012; 149:15941606.

9. Hashimoto R, Yoshida M, Kunugi H, et al. A missense polymorphism (H204R) of a Rho GTPase-activating protein, the chimerin 2 gene, is associated with schizophrenia in men. Schizophr Res. 2005; 73:383-5.

10. Moran LV, Sampath H, Kochunov P, et al. Brain Circuits That Link Schizophrenia to High Risk of Cigarette Smoking. Schizophrenia Bulletin, 2012. Accessed December12, 2012

11. Liu QR, Drgon T, Johnson C, et al. Addiction molecular genetics: 639,401 SNP whole genome association identifies many “cell adhesion” genes. American Journal of Medical Genetics Part B: Neuropsychiatric Genetics. 2006; 141B: 918-925.

12. Desmet FO, Hamroun D, Lalande M, et al. Human Splicing Finder: an online bioinformatics tool to predict splicing signals. Nucleic Acids Research. 2009; 37:e67

13. Johnston MV. Clinical disorders of brain plasticity. Brain and Development. $2004 ; 26: 73-80$.

14. Novak G, Boukhadra J, Shaikh SA, et al. Association of a polymorphism in the NRXN3 gene with the degree of smoking in schizophrenia: A preliminary study. World Journal of Biological Psychiatry. 2009; 10: 929-935.

15. Docampo E, Ribasés $\mathrm{M}$, Gratacos $\mathrm{M}$ et al. Association of Neurexin 3 polymorphisms with smoking behavior. Genes, Brain and Behavior. 2012; 11: 704-711. 
16. Kenney JW, Florian C, Portugal GS, et al. Involvement of Hippocampal Jun-N Terminal Kinase Pathway in the Enhancement of Learning and Memory by Nicotine. Neuropsychopharmacology. 2009; 35:483-492.

17. Notcovich C, Diez F, Tubio MR et al, Histamine acting on H1 receptor promotes inhibition of proliferation via PLC, RAC, and JNK-dependent pathways. Experimental Cell Research. 2010; 316:401-411. 
Table 1 Genotype frequencies of the CHN2 polymorphisms, rs12112301 (IVS5+7C>T), rs3750103 c.611A>G (p.H203R) and rs186911567 c.366G>A (p.S122S) in smokers and control subjects

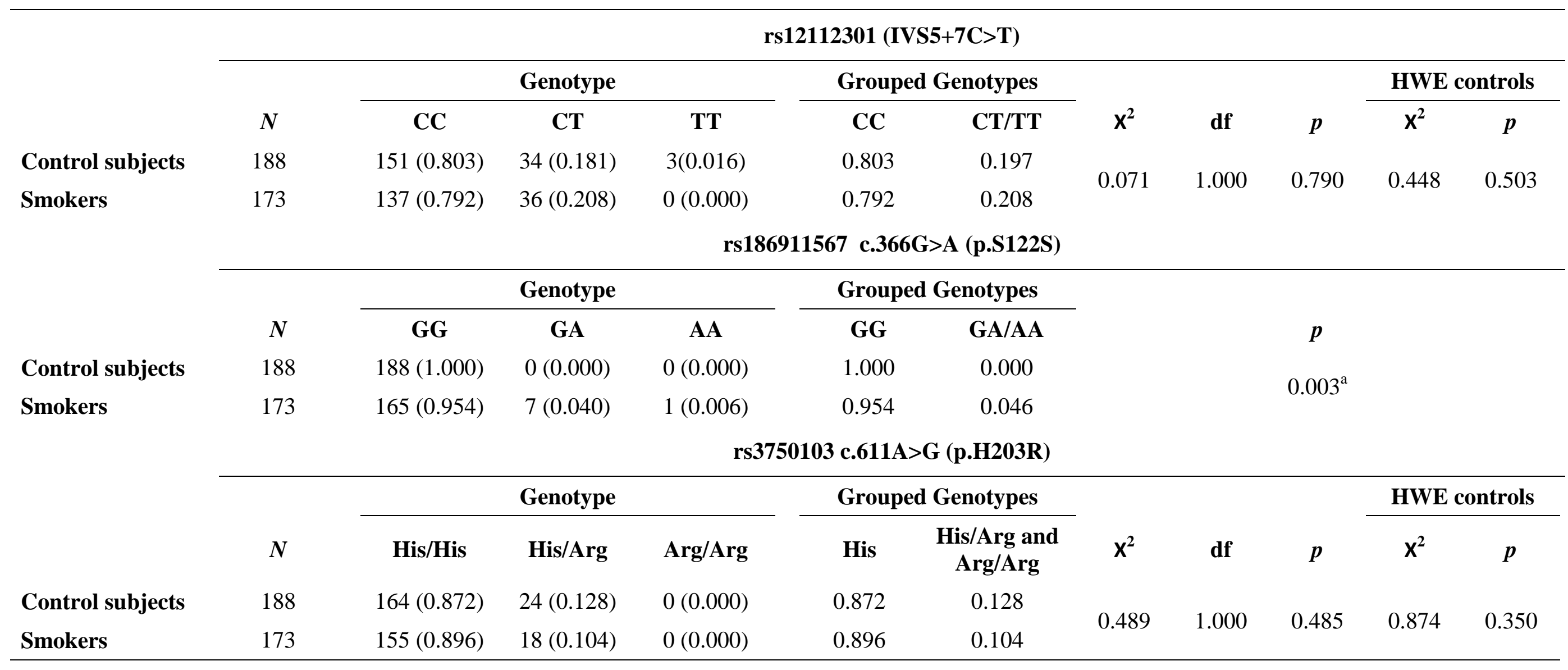

${ }^{\mathrm{a}}$ Fisher's exact test 
Table 2 Genotype frequencies of the CHN2 rs186911567 c.366G>A (p.S122S) polymorphism in smokers with different age and smoking behavior

\begin{tabular}{|c|c|c|c|c|c|c|c|}
\hline & \multirow[b]{2}{*}{$N$} & \multicolumn{3}{|c|}{ Genotype } & \multicolumn{2}{|c|}{ Grouped Genotypes } & \multirow[b]{2}{*}{$p$} \\
\hline & & GG & GA & $\mathbf{A A}$ & GG & GA/AA & \\
\hline \multicolumn{8}{|l|}{ Age (years) } \\
\hline$<65$ & 80 & $79(0.988)$ & $1(0.012)$ & $0(0.000)$ & 0.988 & 0.012 & \multirow{2}{*}{$0.070^{\mathrm{a}}$} \\
\hline$>65$ & 93 & $86(0.925)$ & $6(0.065)$ & $1(0.011)$ & 0.925 & 0.075 & \\
\hline \multicolumn{8}{|l|}{ Cigarettes/day } \\
\hline Up to 20 & 97 & $91(0.938)$ & $5(0.052)$ & $1(0.010)$ & 0.928 & 0.062 & \multirow{2}{*}{$0.468^{\mathrm{a}}$} \\
\hline More than 20 & 76 & $74(0.974)$ & $2(0.026)$ & $0(0.000)$ & 0.974 & 0.026 & \\
\hline \multicolumn{8}{|l|}{ Years smoking } \\
\hline Up to 30 & 78 & $76(0.974)$ & $2(0.026)$ & $0(0.000)$ & 0.974 & 0.026 & \multirow{2}{*}{$0.297^{\mathrm{a}}$} \\
\hline More than 30 & 95 & $89(0.937)$ & $5(0.053)$ & $1(0.011)$ & 0.937 & 0.046 & \\
\hline
\end{tabular}

${ }^{a}$ Fisher's exact test 